\title{
Patient considerations in metastatic colorectal cancer - role of panitumumab
}

This article was published in the following Dove Press journal:

OncoTargets and Therapy

7 April 2017

Number of times this article has been viewed

Jane E Rogers

Pharmacy Clinical Programs, University of Texas MD Anderson

Cancer Center, Houston, TX, USA
Correspondence: Jane E Rogers

Pharmacy Clinical Programs, University of Texas MD Anderson Cancer Center, I5I 5 Holcombe Blvd, Houston, TX 77030, USA

Fax + I 7|3 794 I586

Email jerogers@mdanderson.org
Tel + I 7137943082

Abstract: Epidermal growth factor receptor (EGFR) is overexpressed in many malignancies, including colorectal cancer (CRC), making EGFR an attractive treatment option. Panitumumab and cetuximab, monoclonal antibodies (mAbs) directed at EGFR, are both currently utilized in the management of metastatic CRC (mCRC). Through the development of these agents in $\mathrm{mCRC}$, key issues surrounding each $\mathrm{mAbs}$ use have been revealed. These key issues include negative patient outcome avoidance when determining use, the economic burden with high-cost medication, predictive biomarkers, tumor location, patient geographic location, patient quality of life, and the prevention of debilitating adverse effects. CRC remains a common malignancy, with many of these patients expected to receive targeted therapy, including EGFR mAb therapy. Oncologists must recognize these EGFR mAb factors in order to improve outcomes. This review aims to provide a chronological timeline on the development of panitumumab, clinical pearls, and guidance on the current use of panitumumab in mCRC.

Keywords: receptor, epidermal growth factor, antineoplastic agent, antibodies, monoclonal, colorectal neoplasms

\section{Introduction}

Panitumumab is an $\mathrm{IgG}_{2}$ fully human monoclonal antibody (mAb) targeting the ligand-binding domain of the epidermal growth factor receptor (EGFR). ${ }^{1-6}$ Targeted therapy, including panitumumab, administered alone or in combination with traditional antineoplastics, has been incorporated into metastatic colorectal cancer (mCRC) management for beyond a decade. EGFR and vascular endothelial growth factor (VEGF) represent mCRC targets with current approved United States Food and Drug Administration (FDA) therapy.

\section{Colorectal cancer: incidence and agents}

Colorectal cancer (CRC) remains a common worldwide malignancy, representing $10 \%$ of all new cancer cases globally. ${ }^{7}$ A sizable percentage of CRC patients will be candidates for targeted therapy as $20 \%-25 \%$ present with metastatic disease, while another $20 \%-35 \%$ develop recurrence following curative early stage treatment. ${ }^{5,8-10}$ Metastatic disease has a median overall survival (OS) at $\sim 30$ months. ${ }^{11}$ The mCRC OS improvement over the last few decades, from 10 to 12 months' survival seen with 5-fluorouracil combined with leucovorin modulation to currently beyond 30 months, is multifactorial. ${ }^{11-13}$ Influencing survival are aggressive selective metastatic disease surgical resection and increased localized therapies, largely due to the expanded systemic treatment options available, including therapies targeting EGFR and VEGF. ${ }^{11,14}$ Eleven mCRC antineoplastics are currently the United States Food and Drug Administration (FDA) approved and include, in order of approval, 5-fluorouracil, irinotecan, 


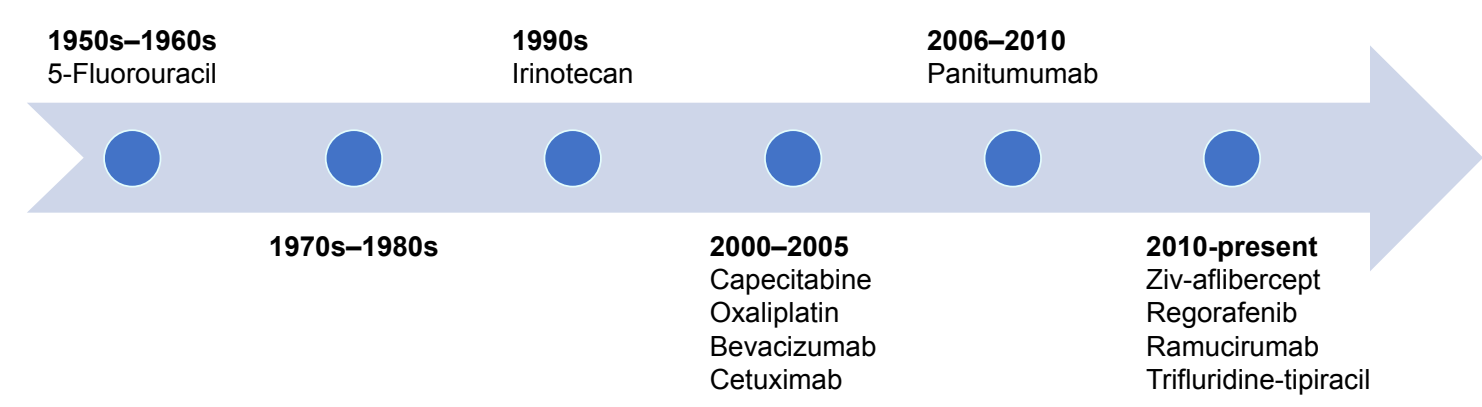

Figure I Timeline mCRC FDA antineoplastic approval.

Abbreviations: $\mathrm{mCRC}$, metastatic colorectal cancer; FDA, United States Food and Drug Administration.

capecitabine, oxaliplatin, bevacizumab, cetuximab, panitumumab, ziv-aflibercept, regorafenib, ramucirumab, and trifluridine-tipiracil (Figure 1).

Cetuximab, an $\mathrm{IgG}_{1}$ chimeric (human; murine) $\mathrm{mAb}$ targeting EGFR, was first FDA approved in February 2004. ${ }^{1,5,15}$ Panitumumab obtained FDA approval shortly after, in September 2006, along with authorization in 2007 with The European Medicines Agency and in 2008 with Health Canada. ${ }^{1,4,16}$ Both EGFR mAbs provide an excellent example of knowledge obtained surrounding cancer targets as patient selection is key and not all patients will derive benefit. Mutations in the rat sarcoma (RAS) oncogene, a downstream pathway to EGFR, have shown to be a predictive biomarker of resistance when cetuximab or panitumumab are given to patients that harbor these mutations. ${ }^{17}$ Despite vast improvements in OS, 5-year OS for mCRC currently remains poor at $13 \%$, revealing the need for further therapies as well as the proper use of currently approved agents. ${ }^{8}$ Oncologists must recognize the correct placement of targeted therapy to improve mCRC outcomes, while avoiding negative outcomes, debilitating adverse effects, and economic concerns. This article aims to provide guidance on panitumumab use in the mCRC setting.

\section{Pharmacokinetics and pharmacodynamics}

EGFR/HER1/ErbB-1, a tyrosine kinase transmembrane receptor belonging to the ErbB family, plays a crucial role in cell proliferation, survival, and differentiation in many malignancies due to EGFR dysregulation., ${ }^{5,16}$ Many ligands, in addition to epidermal growth factor (EGF), are involved in EGFR activation, including transforming growth factor alpha, amphiregulin, betacellulin, epigen, epiregulin, and heparin-binding EGF. These various ligands are capable of binding to the extracellular ligand-binding domain to activate downstream mechanisms for tumor development and proliferation. EGFR activation leads to several signaling pathways, including RAS/RAF/MEK/ERK, PI3CK/AKT/mTOR, Src family kinases, STATs, and PLC $\gamma$-PKC, thus leading to cell survival through tumor growth, angiogenesis, tumor invasion, and migration.

EGFR overexpression is seen in $60 \%-80 \%$ of CRC patients, making EGFR mAb inhibition an attractive treatment option. ${ }^{2,5,16}$ EGFR mAbs, cetuximab and panitumumab, inhibit EGFR by binding to the extracellular ligand-binding domain to compete with these endogenous ligands from binding. ${ }^{1,4,5,15,16}$ By blocking the binding site, inhibition of ligand-induced tyrosine kinase activation can ensue and lead to EGFR cellular internalization and degradation, allowing for cell growth inhibition and apoptosis. There are four extracellular binding domains with domains I and III contributing to ligand binding: both mAbs bind to domain III. ${ }^{16}$ Subtle differences in cetuximab and panitumumab's mechanisms exist. Panitumumab has a higher binding affinity than cetuximab. ${ }^{3,5,16}$ However, cetuximab's mechanism of action is thought to additionally have an immunologic-mediated response with antibody-dependent cellular toxicity due to cetuximab's $\operatorname{IgG}_{1}$ chimeric $\mathrm{mAb}$ structure. Although slight mechanism differences exist between these two agents, clinically cetuximab and panitumumab are currently recommended similarly in mCRC therapy. $1,2,11,14$

Panitumumab is commercially dosed at $6 \mathrm{mg} / \mathrm{kg}$ intravenous over 60-90 minutes (infusion time dependent on dose calculated) every 2 weeks and exhibits nonlinear pharmacokinetics with a half-life of 7.5 days. ${ }^{1-4}$ Panitumumab binds to EGFR, and it is internalized inside the cell. Premedication is not required for administration given the fully human $\mathrm{mAb}$ structure. ${ }^{4}$ Age, concomitant chemotherapy, gender, race, mild-to-moderate renal and liver dysfunction, and EGFR membrane expression showed no impact on panitumumab's pharmacokinetics. ${ }^{1,4,6}$ As with other mAbs and endogenous immunoglobulins, panitumumab, in addition to EGFR degradation, is eliminated by the reticuloendothelial system; therefore, organ dysfunction administration barriers are 
unlikely. Case reports have shown successful use in patients undergoing intermittent hemodialysis with panitumumab and in liver dysfunction. ${ }^{18-20}$ No information on use in human pregnancy is available, and given the mechanism of action of EGFR therapy, this would not be advised. An alternative dosing scheme of panitumumab $9 \mathrm{mg} / \mathrm{kg}$ intravenous every 3 weeks has been reported. ${ }^{21}$ However, for mCRC, a dose of $6 \mathrm{mg} / \mathrm{kg}$ intravenous every 2 weeks remains standard as panitumumab is often administered in combination with traditional chemotherapy regimens that are dosed biweekly. ${ }^{14}$ Panitumumab represents an appealing medication with limited patient barriers, such as organ dysfunction and drugdrug interactions, restricting the drug's use.

\section{History and new updates in $\mathrm{MCRC}$ management \\ Early trial development}

Figure 2 outlines panitumumab development in mCRC, starting in 2005 with initial phase I evaluation. At the 2005 Annual American Society of Clinical Oncology (ASCO) meeting, Weiner et $\mathrm{a}^{22}$ presented data on four panitumumab dosing levels given to 96 solid tumor patients, $40 \%$ having CRC. ${ }^{2,3}$ Dosing ranged from $0.01 \mathrm{mg} / \mathrm{kg}$ to $5 \mathrm{mg} / \mathrm{kg}$ once per week, $6 \mathrm{mg} / \mathrm{kg}$ every 2 weeks, or $9 \mathrm{mg} / \mathrm{kg}$ every 3 weeks. Showing promise in CRC, panitumumab resulted in $12.8 \%$ partial responses, with $23 \%$ stable disease in CRC patients given panitumumab. A phase II safety and efficacy evaluation by Malik et $\mathrm{al}^{23}$ further strengthened the concept for panitumumab in CRC. ${ }^{2,3}$ Panitumumab was dosed at $2.5 \mathrm{mg} / \mathrm{kg}$ monotherapy weekly in refractory mCRC patients divided into EGFR membrane expression cohorts. A total of 148 patients showed a $46.5 \%$ disease control with panitumumab (10\% partial responses and $36.5 \%$ stable disease), a median OS of 9.4 months, and a time to progression of 2.5 months.

\section{Monotherapy for refractory $\mathrm{mCRC}$ : FDA approval}

Panitumumab's initial FDA approval was based on an open-labeled, randomized, multicenter pivotal phase III trial of panitumumab $6 \mathrm{mg} / \mathrm{kg}$ IV every 2 weeks plus best supportive care (BSC) compared to BSC alone in a ( $\geq 1 \%$ EGFR positive) mCRC population by Van Cutsem et al. ${ }^{24}$ The patients had progressed on all standard chemotherapy, including fluoropyrimidines, irinotecan, and oxaliplatin. A total of 463 patients who enrolled during 2004-2005 met the inclusion criteria (231 patients in the panitumumab plus BSC vs 232 patients assigned to BSC) and had an Eastern Cooperative Oncology Group (ECOG) score of $\leq 2$. About 176 patients received panitumumab under crossover protocol. The primary end point of progression-free survival (PFS) was significantly prolonged at 8 weeks in the pantiumumab plus BSC vs 7.3 weeks for BSC alone (HR $=0.54, P=0.0001$ ). At 8 weeks, the PFS rates were $49 \%$ for the pantiumumab arm and $30 \%$ for BSC alone. Objective response rate (ORR) showed similar results to early panitumumab trial

2010-2015

Phase III

panitumumab +

FOLFIRI second-line

Phase III

panitumumab +

FOLFOX frontline

Expanded RAS

testing

**FDA label change promise for

CRC expression

of response

**EMS

approval

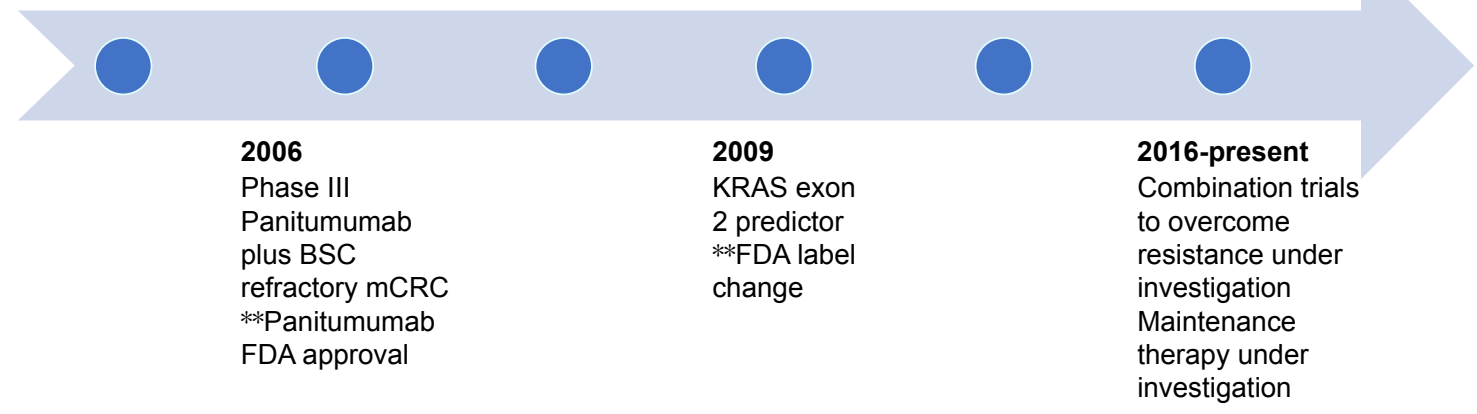

Figure 2 Panitumumab development in $\mathrm{mCRC}$.

Note: **Indicates guideline/package labeling changes.

Abbreviations: CRC, colorectal cancer; BSC, best supportive care; FDA, United States Food and Drug Administration; EGFR, epidermal growth factor receptor; EMS, European Medicines Agency; KRAS, Kristin rat sarcoma; mCRC, metastatic CRC; FOLFIRI, 5-fluorouracil, leucovorin, irinotecan; FOLFOX, 5-fluorouracil, leucovorin, oxaliplatin; RAS, rat sarcoma. 
development, with $10 \%$ partial response and $27 \%$ stable disease in the panitumumab group compared to $0 \%$ partial response and $10 \%$ stable disease for BSC alone. ORR did show statistical significance $(P<0.0001)$. No difference in OS was observed $(\mathrm{HR}=1.00 ; 95 \% \mathrm{CI}$ : 0.82-1.22; $P=0.81)$; however, one would expect such a result given the high amount of crossover. Despite marginal PFS improvement, panitumumab monotherapy was granted FDA approval for EGFR membrane-expressing mCRC refractory to fluoropyrimidines, oxaliplatin, and irinotecan regimens.

\section{EGFR membrane expression and outcomes: does it correlate with outcomes?}

Berlin et $\mathrm{al}^{25}$ conducted a multicenter phase II panitumumab monotherapy trial in ECOG $\leq 2$ refractory mCRC patients (fluoropyrmidines, oxaliplatin, and irinotecan progression) in those with high EGFR membrane expression (EGFR staining $\geq 10 \%$ ). Panitumumab was dosed at $6 \mathrm{mg} / \mathrm{kg} \mathrm{IV}$ every 2 weeks. Ninety-one patients enrolled, of whom $8 \%$ showed a partial response and $21 \%$ stable disease. These outcomes were similar to those of previous evaluations. Mitchell et $\mathrm{al}^{26}$ in the 2007 Annual ASCO Meeting confirmed findings from previous evaluations that panitumumab has antitumor activity in patients regardless of EGFR membrane expression. ${ }^{27}$ The results, stratified according to low (1\%-9\% staining) or negative $(<1 \%)$ EGFR membrane expression, were similar to the Berlin et $\mathrm{al}^{25}$ study, with overall 7\%-9\% partial responses and 30\%-33\% stable disease. Given these findings, EGFR expression was identified as a nonpredictor of EGFR mAb response for $\mathrm{mCRC}$.

\section{Monotherapy for refractory $\mathrm{mCRC}$ : role of Kristen rat sarcoma (KRAS) exon 2 mutations}

Amado et al $^{28}$ provided a prospective-retrospective analysis reexamining the previous panitumumab monotherapy trial by Van Cutsem et al. ${ }^{24}$ The analysis was performed to investigate outcomes related to the presence of KRAS exon 2 mutations. Ninety-two percent were included in the KRAS analysis, with 208 patients in the panitumumab plus BSC arm and 219 patients in the BSC arm. KRAS exon 2 mutations were identified in $43 \%$ of the study population and were equally divided between the two treatment groups. Wild-type KRAS patients had significantly greater PFS, with panitumumab plus BSC showing a median PFS of 12.3 weeks vs 7.3 weeks (HR $=0.45$; 95\% CI: 0.34-0.59). No benefit was seen in those patients with KRAS exon 2 mutations with a median PFS of
7.4 weeks with panitumumab plus BSC compared to 7.3 weeks for BSC (HR $=0.99 ; 95 \%$ CI: 0.73-1.36). Seventeen percent response was seen in the KRAS wild-type group compared to $0 \%$ in the mutant group. Those in the crossover portion of the study showed similar results defined by mutation status, with improvement in PFS seen in KRAS wild-type patients (median PFS 16.4 weeks in KRAS wild type vs 7.9 weeks in KRAS mutant, $\mathrm{HR}=0.32 ; 95 \% \mathrm{CI}: 0.22-0.45$ ). Amado et $\mathrm{al}^{28}$ helped define the predictive role that KRAS exon 2 mutations play in EGFR mAb therapy. In July 2009, the FDA approved revisions to EGFR mAb class labeling to reflect KRAS exon 2 mutations as possible predictive biomarkers for efficacy of anti-EGFR therapy.

\section{Panitumumab vs cetuximab: monotherapy for refractory $m C R C$}

A Study of Panitumumab Efficacy and Safety Compared to Cetuximab (ASPECCT) trial was the first head-to-head trial comparing the two EGFR mAbs. ${ }^{29}$ ASPECCT was an openlabeled, randomized, multicenter phase III noninferiority trial of wild-type KRAS exon 2 mCRC chemotherapy refractory population with progression on irinotecan-based and oxaliplatin-based therapy, and a thymidylate synthase inhibitor. Patients had an ECOG $<2$. Dosing of panitumumab was $6 \mathrm{mg} / \mathrm{kg}$ IV every 2 weeks compared to cetuximab $400 \mathrm{mg} / \mathrm{m}^{2}$ loading dose followed by $250 \mathrm{mg} / \mathrm{m}^{2}$ weekly. A total of 999 patients enrolled during 2010-2012 met the inclusion criteria, 499 of whom were in the panitumumab group and 500 in the cetuximab group. OS was the primary end point and showed a median OS of 10.4 months (95\% CI: 9.4-11.6 months) with panitumumab and 10 months with cetuximab (95\% CI: 9.3-11.0 months), HR =0.97 (95\% CI: 8.4-1.11). Twenty-two percent (95\% CI: 18.4-26) showed an objective response in the panitumumab group and 19.8\% $(95 \%$ CI: $16.3-23.6)$ in the cetuximab group $(\mathrm{HR}=1.15 ; 95 \% \mathrm{CI}$ : 0.83-1.58). As the ASPECCT trial revealed, clinically these agents are noninferior when given for refractory mCRC.

\section{Second-line therapy in combination with chemotherapy}

Peeters et $\mathrm{al}^{30}$ conducted an open-label, randomized, multicenter phase III trial comparing panitumumab plus 5-fluorouracil, leucovorin, irinotecan (FOLFIRI) compared to FOLFIRI alone in mCRC population treated with progression on one prior therapy. Patient had an ECOG $<2$. Panitumumab was given at $6 \mathrm{mg} / \mathrm{kg}$ IV every 2 weeks. A total of 1,186 patients were enrolled in 2006-2008, with 591 patients in the panitumumab combination arm and 595 patients in the 
FOLFIRI-alone group. KRAS status was confirmed in $91 \%$ of the patients, with $55 \%$ wild type and $45 \%$ mutant. In the wild-type KRAS group, panitumumab added to FOLFIRI significantly improved PFS, with a median PFS of 5.9 months compared to 3.9 months ( $\mathrm{HR}=0.73$; 95\% CI: $0.59-0.90$; $P=0.004)$. A nonsignificant trend toward increased OS was observed in the wild-type group (median OS was 14.5 months vs 12.5 months, $\mathrm{HR}=0.85,95 \% \mathrm{CI}: 0.70-1.04 ; P=0.12$ ), and a $35 \%$ improvement in the response rate against $10 \%$ was observed with the addition of panitumumab. Mutant KRAS patients showed no benefit with the addition of panitumumab in PFS (5 months vs 4.9 months, $P=0.14$ ), OS (OS 11.8 vs 11.1 months), or response rate (13\% vs $14 \%)$. Further confirming the importance of KRAS status, Peeters et $\mathrm{al}^{30}$ showed panitumumab in combination with FOLFIRI is an option in the second-line setting for KRAS exon 2 wildtype patients.

\section{First-line therapy in combination with chemotherapy}

Hecht et $\mathrm{al}^{31}$ examined the role of dual biologics with chemotherapy in an open-labeled, randomized, multicenter phase III trial that assigned $\mathrm{mCRC}$ patients to either bevacizumab plus 5-fluorouracil, leucovorin, and oxaliplatin (FOLFOX) or bevacizumab plus FOLFIRI to \pm panitumumab. ${ }^{31}$ Patients were treatment naïve. After a preplanned analysis, the trial was halted due to significantly inferior PFS in the dual biologic group in the FOLFOX cohort. Confirmed additionally with the CAIRO2 cetuximab trial, dual biologics were shown to be unfavorable and not recommended. ${ }^{27}$

The PRIME study, carried out by Douillard et al, ${ }^{32}$ looked at panitumumab in the frontline setting. ${ }^{32}$ A phase III, openlabeled, randomized, multicenter trial compared the addition of panitumumab $6 \mathrm{mg} / \mathrm{kg}$ IV every 2 weeks with FOLFOX4 vs FOLFOX4 alone. Patients had an ECOG $\leq 2$. EGFR expression and KRAS status were not required at entry. A total of 1,183 patients were enrolled during 2006-2008, with 593 patients in the panitumumab arm and 590 patients in the control arm. Forty percent of patients were found to have KRAS exon 2 mutant tumors. PFS improvement was shown in the wild-type KRAS exon 2 group, with a median 9.6 months (95\% CI: 9.2-11.1 months) compared to 8.0 months (95\% CI: 7.5-9.3 months) in the FOLFOX4 arm alone. OS favored the panitumumab arm with a 4.2-month improvement for wild-type KRAS patients; however, this did not reach statistical significance (23.9 months compared to 19.7 months, $P=0.072$ ). In the mutant KRAS exon 2 group, unfavorable results were seen with the addition of panitumumab, with a median PFS of 7.3 months (95\% CI: 6.3-8.0 months) compared to 8.8 months (95\% CI: 7.7-9.4 months) for FOLFOX4, with OS additionally showing poorer survival in the panitumumab arm but not reaching statistical significance (median OS 15.5 months compared to 19.3 months, $P=0.068$ ).

\section{Update of prime study: beyond KRAS exon 2 mutations}

RAS mutations significant for mCRC encompass mutations in KRAS and neuroblastoma RAS (NRAS). ${ }^{11-14,33,34}$ As evident through previous evaluations of EGFR $\mathrm{mAb}$ therapy in $\mathrm{mCRC}$, KRAS exon 2 mutations are a negative predictive marker for EGFR inhibitor therapy and lead to worse outcomes when given these agents. KRAS exon 2 mutations are seen in $40 \%$ of CRC patients, and, as evidenced by recent publications, another $10 \%-20 \%$ of wild-type KRAS exon 2 (codon 12 and 13) patients will harbor mutations in KRAS exons 3 (codon 61) or 4 (codon 117 and 146) or in NRAS exons 2 (codon 12 and 13), 3 (codon 61), or 4 (codon 117 and 146).

Douillard et $\mathrm{al}^{33}$ performed a prospective-retrospective analysis of the PRIME trial to evaluate the outcomes and incidence of these mutations. Seventeen percent of patients were found to have additional RAS mutations outside of KRAS exon 2. Expanded RAS testing showed shorter PFS and OS in the RAS-mutant population when given panitumumab (median PFS of 7.3 months vs 8.7 months, $P=0.008$; median OS of 15.5 months vs 18.7 months, $P=0.04$ ). Updates with the wild-type RAS group showed further improvement in outcomes with the addition of panitumumab to FOLFOX4 (PFS of 10.1 months vs 7.9 months, $P=0.004$; and OS of 26.0 months vs 20.2 months, $P=0.04$ ). These results of expanded RAS testing are additionally confirmed with updates of the FIRE-3 trial with cetuximab. ${ }^{34}$ In 2015, FDA approved labeling changes for RAS for panitumumab and cetuximab.

\section{Expanded RAS testing: economically favorable}

The current and future economic burden of cancer therapy is evident given prolonged survival and the growing number of newly approved high-cost agents. In the United States, CRC spending is estimated to double by the year 2020, with an estimated total expenditure of $\$ 5.19$ billion in the initial phase of care (first 12 months after diagnosis), \$3.57 billion in the continuing phase (between the initial and the last phase), and $\$ 5.27$ billion in the last phases of care (last 12 months of life). ${ }^{35}$ High drug costs of the 11 antineoplastics utilized in CRC are contributing to this economic burden. Cost, unfavorable outcomes, and toxicity are the reasons to 
clearly identify the role of targeted therapy and the patients likely to benefit. Expanded RAS testing has clearly shown implications for accurate $\mathrm{mCRC}$ treatment. A recent analysis examined the economic implications of expanded RAS testing and identified potential annual drug cost savings of $\$ 184$ million when these $18 \%$ additional RAS patients are identified using various potential prices for RAS testing. ${ }^{36}$ A comparison of testing laboratory fee amounts (example KRAS =\$197) with the cost of each EGFR mAb and the potential duration of therapy points to the economic benefits of testing.

\section{Update: role of primary tumor location and targeted therapy}

mCRC is clearly a heterogenous disease, as revealed by many factors, including epidemiology, presentation of disease, aggressive nature seen in certain cases, age, and mutational characteristics correlating with prognosis and response to therapy. Two recent retrospective analyses have revealed the most compelling evidence examining the primary tumor location for heterogeneity with regard to outcomes and treatment..$^{37,38}$ Tejpar et $\mathrm{al}^{37}$ retrospectively looked at RAS wild-type mCRC patients from the CRYSTAL and FIRE-3 trials. The CRYSTAL and FIRE-3 trials utilized cetuximab in combination with FOLFIRI in the first-line setting with different comparator arms (CRYSTAL comparator = FOLFIRI; FIRE-3 comparator $=$ bevacizumab + FOLFIRI). Tumors located in the splenic flexure, descending colon, sigmoid colon, or rectum were classified as left-sided tumors, while those present in the appendix, cecum, ascending colon, hepatic flexure, or transverse colon were placed in the right-sided group. Superiority in PFS and OS was found in patients with left-sided tumors compared to those with right-sided tumors.

At the 2016 annual ASCO meeting, Venook et al ${ }^{38}$ presented an analysis of retrospectively evaluated data from the CALGB/SWOG 80405 clinical trial. The CALGB/SWOG 80405 trial examined first-line chemotherapy (FOLFOX or FOLFIRI) + cetuximab compared to first-line chemotherapy (FOLFOX or FOLFIRI) + bevacizumab. Right-sided tumors were defined as cecum to the hepatic flexure, and those classified as left-sided were from the splenic flexure to the rectum. Key findings revealed a difference in survival and targeted therapy dependent on tumor location. OS was longer in those with left-sided mCRC compared to right-sided mCRC (34.2 months vs 19.4 months, $\mathrm{HR}=1.56$; 95\% CI: 1.32-1.84). The cetuximab arm showed in those with left-sided tumors an OS of 37.5 months compared to
16.4 months in the right-sided location arm $(\mathrm{HR}=1.97$; 95\% CI: 1.56-2.48). The bevacizumab arm revealed an OS for left-sided tumors of 32.1 months compared to 24.5 months $(\mathrm{HR}=1.26$; 95\% CI: 1.00-1.58). Given this dramatic difference in the EGFR mAB group with tumor location, national guidelines have since been updated to reflect tumor location specifically to reserve frontline EGFR $\mathrm{mAb}$ therapy for left-sided RAS wild-type mCRC only. ${ }^{14}$ If RAS testing results are available at initial diagnosis and an EGFR mAb therapy is being considered, tumor location should be a consideration when deciding upfront systemic therapy.

\section{Panitumumab: current place in therapy}

Expanded RAS mutational testing is recommended/ mandatory for all mCRC patients at diagnosis currently by the National Comprehensive Cancer Network (NCCN), ASCO, and the European Society of Medical Oncology due to the known negative predictive marker for EGFR mAb therapy. ${ }^{11,14,39}$ Guidelines, as described above, do not differentiate or recommend a preference over cetuximab or pantiumumab when an EGFR mAb is recommended. For first-line therapy, pantiumumab and cetuximab are options listed in combination with FOLFOX or FOLFIRI chemotherapy in RAS wild-type only patients with sidedness consideration. ${ }^{11,14}$ Currently, neither guideline states a preference for biologic therapy (bevacizumab or an EGFR mAb) in combination with chemotherapy in these RAS wild-type patients in which the goal is prolonged survival with the exception of recent update in the NCCN guidelines recommending left-sided tumors only for upfront EGFR mAb therapy. If an EGFR $\mathrm{mAb}$ therapy was not provided frontline for RAS wild-type patients, second-line therapy options include cetuximab or panitumumab in combination with FOLFIRI or irinotecan. If EGFR mAb therapy was not provided first- or second-line, cetuximab or panitumumab monotherapy or in combination with irinotecan are options for RAS wild-type patients in the third-line setting.

\section{Adverse effects}

Targeted therapy has presented new challenges to the oncology health care team regarding toxicity prevention and management, revealing nontraditional antineoplastic adverse effects. EGFR present in normal tissues of the epithelial, hair follicle, and gastrointestinal tract lead to the adverse effects seen with these agents. Counseling and preventive measures along with aggressive management are key in avoiding severe EGFR inhibitor toxicity and are listed in Table 1. 
Table I Toxicity prevention and management

\begin{tabular}{|c|c|c|}
\hline Toxicity & Prevention/monitoring & Management \\
\hline $\begin{array}{l}\text { Papulopustular } \\
\text { rash }\end{array}$ & $\begin{array}{l}\text { Mild soaps and lotions } \\
\text { Avoid alcohol- and perfume-based soaps and lotions } \\
\text { Avoid skin friction (shaving; strong rubbing with a towel) } \\
\text { Avoid hot showers; wash with lukewarm water } \\
\text { Sun protection } \\
\text { Aggressive, bland, thick emollient (urea based) } \\
\text { Limit exposure to severe weather } \\
\text { Prophylactic: doxycycline or minocycline }\end{array}$ & $\begin{array}{l}\text { Topical antibiotics (clindamycin; erythromycin) } \\
\text { Oral antibiotics (doxycycline; minocycline) } \\
\text { Low potency steroids for face; medium-potency steroids } \\
\text { for body } \\
\text { Severity may require temporary cessation or dose adjustment }\end{array}$ \\
\hline Xerosis & $\begin{array}{l}\text { Mild soaps and lotions } \\
\text { Avoid alcohol- and perfume-based soaps and lotions } \\
\text { Avoid hot showers, long showers, long baths } \\
\text { Wash with lukewarm water } \\
\text { Aggressive, bland, thick emollient (containing urea, } \\
\text { colloidal oatmeal, and petroleum-based creams, zinc oxide) } \\
\text { Limit exposure to severe weather } \\
\text { Sun protection }\end{array}$ & $\begin{array}{l}\text { Aggressive, bland, thick emollient use (alternative emollient) } \\
\text { Reinforce prevention strategies } \\
\text { Severity may require topical medium- to high-potency steroids }\end{array}$ \\
\hline Fissures & $\begin{array}{l}\text { Aggressive, bland, thick emollient use } \\
\text { Avoid skin irritants/protective coverings/avoid friction }\end{array}$ & $\begin{array}{l}\text { Zinc oxide, ferric subsulfate, silver nitrate, cyanoacrylate glue } \\
\text { to promote healing } \\
\text { Antiseptic soaks to prevent infection (vinegar: water; diluted } \\
\text { bleach: water) }\end{array}$ \\
\hline Pruritus & $\begin{array}{l}\text { Mild soaps and lotions } \\
\text { Avoid alcohol- and perfume-based soaps and lotions } \\
\text { Aggressive, bland, thick emollient }\end{array}$ & $\begin{array}{l}\text { Topical or oral antipruritics } \\
\text { Cold compresses } \\
\text { Medium- to high-potency steroids } \\
\text { Steroid shampoo for scalp (fluocinonide } 0.05 \% \text {, clobetasol foam) } \\
\text { Pregabalin/gabapentin }\end{array}$ \\
\hline Paronychia & $\begin{array}{l}\text { Avoid tight shoes and skin manipulation } \\
\text { Cotton gloves for hand protection } \\
\text { Keep nails clean, dry, and trim } \\
\text { Emollient application regularly } \\
\text { Avoid contact with skin irritants }\end{array}$ & $\begin{array}{l}\text { Cushing inserts for affected toes } \\
\text { Antiseptic soaks (vinegar: water; diluted bleach: water) } \\
\text { Mild- to medium-potency topical steroids for inflammation } \\
\text { without infection } \\
\text { Culture and antimicrobials for bacterial or fungal superinfection } \\
\text { Severity (grade } \geq 2 \text { ) may require temporary cessation or dose } \\
\text { adjustment of EGFR mAb }\end{array}$ \\
\hline Hypomagnesemia & $\begin{array}{l}\text { Magnesium level at baseline, during therapy, and continuing } \\
\text { at least } 8 \text { weeks after discontinuation } \\
\text { Recommend diligent monitoring for higher risk patients } \\
\text { (elderly, cardiac comorbidities, longer duration of therapy) }\end{array}$ & $\begin{array}{l}\text { Oral magnesium supplements } \\
\text { Intravenous magnesium replacement }\end{array}$ \\
\hline
\end{tabular}

Abbreviations: EGFR, epidermal growth factor receptor; $\mathrm{mAB}$, monoclonal antibody.

\section{Skin toxicities}

Skin toxicities, including rash, xerosis, photosensitivity, paronychia, and fissures, represent the most common adverse effects seen with these agents, with a papulopustular rash being the most frequently reported. ${ }^{4,15,40-45}$ Seen in $50 \%-95 \%$ of patients receiving EGFR mAbs, the papulopustular rash is not only the most common toxicity but is also the earliest in presentation, occurring as early as 2 days after initiation and peaking, most often by the second week of therapy. The rash may present as late as 6 weeks after initiation. Termed the acneiform rash, the EGFR mAb rash differs from acne in that comodones are not often present. Initial symptoms are edema and erythema that progress to papules, nodules, and pustules on the face, neck, chest, back, scalp, and proximal upper extremities, which by the fourth week often crust over. ${ }^{40,41}$ Pruritus, swelling, and pain are common symptoms that accompany this toxicity.

EGFR is present in the basal and suprabasal layers of the epidermis, the outer layers of the hair follicle, and sebaceous glands. ${ }^{61-45}$ When EGFR inhibition occurs, keratinocyte differentiation is altered in the epithelium and hair follicle, leading to obstruction, inflammation, and bacterial superinfection. While on cetuximab or panitumumab, this toxicity is quite cumbersome for patients due to persistent symptoms, which often wax and wane over time. Therefore, proactive prevention and management should be common practice. The rash does resolve after cessation of therapy $\sim 8$ weeks after discontinuation.

The Skin Toxicity Evaluation Protocol with Panitumumab (STEPP) trial presented the current basis of being preventive 
rather than reactive in controlling the acneiform rash. ${ }^{46}$ The STEPP trial was an open-label phase II trial of 95 patients that examined whether the difference between grade $\geq 2$ panitumumab skin toxicities existed if patients were given preemptive treatment (48 patients) vs reactive treatment after the skin toxicity developed (47 patients) over a 6-week period. The preemptive regimen started 1 day prior to the first panitumumab administration and continued for 6 weeks. This regimen included skin moisturizer applied to the face, hands, feet, neck, back, and chest every morning; sunscreen to areas exposed; topical steroids ( $1 \%$ hydrocortisone cream) applied to the face, hands, feet, neck, back, and chest at bedtime; and doxycycline $100 \mathrm{mg}$ twice per day. The reactive treatment regimen consisted of any treatments the investigator deemed necessary for management and could be given at any time point during the 6 weeks. The trial showed that patients on a preventive regimen had a decrease in $\geq$ grade 2 toxicities, with $29 \%$ in the preemptive group compared to $62 \%$ in the reactive group. Since the STEPP trial, several counseling and preventive techniques have been proposed and are listed in Table 1.

Discussed earlier was the RAS mutation serving as a biomarker predictive of EGFR inhibitor use failure, but some studies suggest that a clinical marker of response lies in the severity of rash. Previous studies with cetuximab and panitumumab generate this hypothesis, including the EVEREST study, by Van Cutsem et al, ${ }^{47}$ which investigated cetuximab dose escalation in those with no or mild skin reactions by the third week of standard dose therapy. ${ }^{48}$ Although compelling, oncologists must discuss this potential with patients cautiously as those without a rash may find this disheartening, while those with a rash will expect positive results. Further, current guidelines still warrant diagnostic imaging to determine response to therapy with these agents and not to rely on the degree of rash.

\section{Skin toxicities: quality of life}

A recent review examined the phase III randomized trials discussed above looking at panitumumab monotherapy in refractory mCRC, panitumumab plus FOLFIRI in the second-line setting, and panitumumab plus FOLFOX in the first-line setting to determine quality of life (QoL) affected by skin toxicity. ${ }^{49}$ EuroQoL 5-domain health state index score (HSI) was utilized along with an overall health rating (OHR). EuroQoL 5 domain HSI evaluates 5 items, including mobility, self-care, usual activities, pain/discomfort, and anxiety/depression, to determine a score from -0.594 to 1.0 , with the higher score indicating better health.
The OHR uses a visual analog scale of $0-100(0=$ worst health state imaginable; $100=$ best health state imaginable). From baseline to discontinuation, there were no statistical differences between the panitumumab group and the comparator arms in all three studies. Skin toxicity regardless of severity level had a similar impact on QoL. Thaler et $\mathrm{l}^{50}$ evaluated skin toxicity in relation to QoL in a phase II frontline trial of panitumumab plus FOLFIRI compared to FOLFIRI alone. Two instruments, the EuroQoL 5-domain HSI and the European Organization for Research and Treatment of Cancer (EORTC) QLQ-C30, evaluated multiple factors such as physical, cognitive, emotional, social, fatigue, pain, nausea/vomiting, global health status/QoL scale, and a perceived financial impact. QoL outcomes were found to be similar at baseline and 8 weeks after the completion of the study in this evaluation.

The STEPP trial evaluating preemptive compared to reactive skin toxicity treatment assessed QoL using the Dermatology Life Quality Index (DLQI) at weeks 2-7 and at week 13 or $14 .{ }^{46}$ The DLQI consists of 10 questions with a score scale of $0-30$, with higher scores indicating more QoL impairment. Questions asked focus on symptoms, emotion, interference with working and social outings, and activities avoided. The results indicated that QoL was less impaired in the preemptive group as the change in score from baseline to week 3 in the preemptive group was 1.3 points compared to the reactive group of 4.2 points. These evaluations reveal that panitumumab may have limited impact on QoL while favoring a preemptive skin toxicity management.

\section{Paronychia}

Paronychia, inflammation of the nail folds of the fingers and toes, particularly the first digits, occurs later in EGFR inhibitor therapy, with a typical onset of 2-6 months after initiation. ${ }^{41,43-45,51,52}$ EGFR inhibition is believed to directly inhibit keratinocytes in the nail matrix, leading to this adverse effect. Occurring in $\sim 20 \%$ of patients on cetuximab or panitumumab, paronychia is a less common toxicity than previously discussed skin toxicities. ${ }^{4,15}$ Nail changes can initially be mild and asymptomatic, but paronychia can lead to symptomatic, painful, and severe complications, including bacterial and fungal superinfection, pyogenic granuloma, and ingrown nails. ${ }^{41,43-45,51,52}$

Clear evidence-based guidelines for prevention and management are lacking. Prevention strategies based on expert opinion and consensus focus on preventive measures such as counseling on the importance of wearing 
comfortable well-fitting shoes; keeping nails trim, clean, and dry; avoidance of bare feet; cushioning inserts in shoes for comfort of the affected nails; petroleum jelly application; and wearing cotton-lined gloves to avoid friction for hand protection. ${ }^{41,43-45,51,52}$ As mentioned, paronychia places a patient at high risk for developing bacterial and fungal superinfections; therefore, the use of antiseptic soaks with a vinegar:water ratio of 1:10 daily along with a mild-to-moderate topical corticosteroid should be advised when inflammation appears without signs of infection. Staphylococcus aureus infections appear to develop acutely, while chronic superinfections are associated with Candida albicans. ${ }^{41}$ Pseudomonas and Enterococcus have additionally been isolated. When superinfection appears evident due to purulent discharge and collection, culture and appropriate antimicrobials should follow. Topical/systematic antibiotics such as topical mupirocin, oral antistaphylococcal penicillin or first-generation cephalosporin, and oral doxycycline have been utilized. Comorbidities such as diabetes may require clindamycin and amoxicillin/clavulanate, and methicillin-resistant $S$. aureus isolates might require sulfamethoxazole/trimethoprim.

\section{Hypomagnesemia}

EGFR regulation at the distal tubules of the kidneys gives way to another common adverse effect of hypomagnesemia seen with panitumumab and cetuximab. EGFR has been shown as a regulatory factor for transient receptor potential cation channel, subfamily melastatin, member 6 (TRPM6). ${ }^{53,54}$ TRPM6 is responsible for active magnesium reabsorption in the distal tubules, and EGFR inhibition has been linked to insufficient TRPM6 function, thus leading to magnesium wasting. Unlike skin toxicities associated with EGFR therapy, hypomagnesemia does not have a clear chronological timing of onset and can occur days to months after therapy initiation. Hypomagnesemia does appear to be progressive, with increased severity seen with longer duration of therapy.

The incidence of hypomagnesemia reported in the prescribing information is seen more with panitumumab $(30 \%$ any grade) compared to cetuximab ( $14 \%$ any grade). ${ }^{4,15}$ Further reports show a higher incidence for EGFR mAb-induced hypomagnesemia with reports as high as $90 \%-100 \%$ all grades with grade $3 / 4$ hypomagnesemia reported at $6 \%-47 \%$ dependent on duration of therapy with $>6$ months of therapy showing a higher severity incidence. ${ }^{53,54}$ Patients often will be asymptomatic. Given the asymptomatic presentation and to avoid severe clinical manifestations of hypomagnesemia, including cardiac arrhythmias and neurocognitive dysfunction, close monitoring of magnesium levels is recommended per prescribing information during EGFR mAb administration and for at least 8 weeks following the cessation of these agents. ${ }^{4,15}$

Guidelines for management during therapy have been less described compared to other adverse effects seen with these agents, and replacement as needed is recommended per prescribing recommendations. ${ }^{4,15}$ Magnesium replacement strategies, including oral magnesium and intravenous magnesium, have been evaluated with a lack of sustainable replacement due to limitations with their use. Issues surrounding oral magnesium are poor absorption and diarrhea, which can be burdensome to patients with a primary CRC malignancy. Intravenous magnesium replacement leads to inconvenience, with longer infusion times for patients, additive days and cost with home health administration, or additional trips to infusion centers. Given these barriers, hypomagnesemia remains an adverse effect in need of a strong and effective preventive and management strategy. Close monitoring and aggressive replacement should be common practice, with diligent monitoring in those patients reporting diarrhea or muscular weakness or cramping, on long duration of therapy ( $>6$ months), in the elderly, and in patients with cardiac comorbidities.

\section{Infusion-related reactions}

Infusion-related reaction rates hold differences among panitumumab and cetuximab given their structural backbone, human vs chimeric ( $3 \%$ panitumumab vs $15 \%-21 \%$ cetuximab). ${ }^{4,15,55,56}$ As mentioned earlier, panitumumab is given at the same rate with each infusion without the use of premedications. ${ }^{4}$ Cetuximab requires the use of an H-1 antagonist (diphenhydramine) given 30 minutes prior to exposure along with a prolonged infusion for the first cycle of therapy. ${ }^{15}$ An area of high cetuximab hypersensitivity likelihood exists in the southeastern region of the United States. ${ }^{55,56}$ The pathogenesis for this higher hypersensitivity rate is related to the presence of preexisting $\operatorname{IgE}$ antibodies to galactose- $\alpha-\mathrm{I}-3$, galactose, a component added during cetuximab production during the chimeric process. ${ }^{6,56}$ Given the high likelihood in this area and the choice of an agent in a malignancy with approval of both agents, an oncologist might consider the use of the fully human $\mathrm{mAb}$ as compared to its chimeric counterpart in this higher risk area. In patients that develop a cetuximab infusion reaction, EGFR mAb rechallenge with panitumumab or cetuximab with modifications have been reported. ${ }^{57,58}$ 
The use of EGFR mAb and tyrosine kinase inhibitors has shown common adverse effects that allow for management programs to be in place. Preemptive management of the common acneform rash should be discussed and presented to each patient at the start of EGFR mAb therapy. Electrolyte balance regarding magnesium should be corrected prior to initiation and followed routinely with consideration of a hypomagnesemia treatment protocol. Finally, routine follow-up with a history and physical while the patient is on therapy should include the examination of nail beds for the presence of paronychia to prevent substantial infections.

\section{Conclusion and future directions}

$\mathrm{mCRC}$ treatment with the addition of biologic agents, including panitumumab, has made dramatic improvements in the OS, which has now reached beyond 30 months. However, as evidenced by a reduced 5-year OS, continued improvement in mCRC management is essential, with future directions examining the heterogeneity of $\mathrm{mCRC}$ and the mechanisms of resistance seen with current therapies. Resistance patterns, both primary and acquired, are a clear impediment in the use of EGFR mAb therapy. ${ }^{5}$ Areas currently under investigation are the additional role of rare RAS mutations, BRAF mutations, PIK3CA mutations and loss of PTEN, and HER2 amplification. Investigations into these resistance patterns are often utilizing combination targeted therapy such as PI3K-mTOR inhibitors, BRAF inhibitors, and MEK inhibitors along with EGFR mAbs. Further, evaluations examining tumor location heterogeneity are likely to be a focus of future investigation. While, clearly, future therapies are needed, a goal of oncology care should always be to make antineoplastics tolerable. Further trials continue to look at the role of maintenance EGFR mAb monotherapy in the hope of avoiding adverse effects of chronic traditional chemotherapy such as fatigue, myelosuppression, and neuropathy. In the case of EGFR mAb therapy, the oncology health-care team must remain proactive, with skin toxicity management and prevention, diligent magnesium monitoring with magnesium replacement protocols in place, and in the avoidance of acute and chronic paronychial infections.

\section{Disclosure}

The author reports no conflicts of interest in this work.

\section{References}

1. Giusti RM, Shastri KA, Cohen MH, Keegan P, Pazdur R. FDA drug approval summary: panitumumab (Vectibix). Oncologist. 2007;12(5): 577-583.

2. Kim GP, Grothey A. Targeting colorectal cancer with human anit-EGFR monoclonal antibodies: focus on panitumumab. Biologics. 2008;2(2): $223-228$.
3. Williams KJ, Lockhart AC. Targeting colorectal cancer with anti-epidermal growth factor receptor antibodies: focus on panitumumab. Onco Targets Ther. 2009;18(2):161-170.

4. Vectibix [package insert]. Thousand Oaks, CA: Amgen, Inc; 2015. Available from: http://pi.amgen.com/ /media/amgen/repositorysites/ pi-amgen-com/vectibix/vectibix_pi.ashx. Accessed December 27, 2016.

5. Sforza V, Martinelli E, Ciardiello F, et al. Mechanisms of resistance to anti-epidermal growth factor receptor inhibitors in metastatic colorectal cancer. World J Gastroenterol. 2016;22(28):6345-6361.

6. Yang BB, Lum P, Chen A, et al. Pharmacokinetic and pharmacodynamic perspectives on the clinical drug development of panitumumab. Clin Pharmacokinet. 2010;49(11):729-740.

7. Torre LA, Bray F, Siegel RL, Ferlay J, Lortet-Tieulent J, Jemal A. Global cancer statistics, 2012. CA Cancer J Clin. 2015;65(2):87-108.

8. National Cancer Institute. Surveillance, Epidemiology, and End Results Program. SEER facts and figures. Colorectal Cancer. Available from: https://seer.cancer.gov/statfacts/html/colorectal.html. Accessed December 7, 2016.

9. Schmoll HJ, Van Custem E, Stein A, et al. ESMO consensus guidelines for management of patients with colon and rectal cancer: a personalized approach to clinical decision making. Ann Oncol. 2012; 23(10):2479-2516.

10. André T, Boni C, Navarro M, et al. Improved overall survival with oxaliplatin, fluorouracil, and leucovorin as adjuvant treatment in stage II or III colon cancer in the mosaic trial. J Clin Oncol. 2009;27(19): 3109-3116.

11. Van Custem E, Cervantes A, Adam R, et al. ESMO consensus guidelines for the management of patients with metastatic colorectal cancer. Ann Oncol. 2016;27(8):1386-1422.

12. Thirion P, Michiels S, Pignon JP, et al. Modulation of fluorouracil by leucovorin in patients with advanced colorectal cancer: an updated meta-analysis. J Clin Oncol. 2004;22(18):3766-3775.

13. Meta-analysis Group in Cancer, Piedbois P, Rougier P, Buyse M, et al. Efficacy of intravenous continuous infusion of fluorouracil compared with bolus administration in advanced colorectal cancer. Clin Oncol. 1998;16(1):301-308.

14. National Comprehensive Cancer Network. NCCN Guidelines: Colon Cancer. Version 2.2017. Available from: https://www.nccn.org/ professionals/physician_gls/pdf/colon_blocks.pdf. Accessed March 23, 2017.

15. Erbitux [package insert]. Indianapolis, IN: Eli Lilly and Company; 2016. Available from: http://pi.lilly.com/us/erbitux-uspi.pdf. Accessed December 27, 2016

16. Yewale C, Baradia D, Vhora I, Patil S, Misra A. Epidermal growth factor receptor targeting in cancer: a review of trends and strategies. Biomaterials. 2013;34(34):8690-8707.

17. Peeters M, Karthaus M, Rivera F, Terwey JH, Douillard JY. Panitumumab in metastatic colorectal cancer: the importance of tumour RAS status. Drugs. 2015;75(7):731-748.

18. Kobayashi M, Endo S, Hamano Y, et al. Successful treatment with modified FOLFOX6 and panitumumab in a cecal cancer patient undergoing hemodialysis. Intern Med. 2016;55(2):127-130.

19. Bolonesi RM, Rogers JE, Shureiqi I. A case report-treatment of metastatic colorectal cancer in a patient on hemodialysis. $J$ Gastrointest Cancer. 2014;45(Suppl 1):161-165.

20. Krens LL, Baas JM, de Jong FA, Guchelaar HJ, Gelderblom H. Pharmacokinetic of panitumumab in a patient with liver dysfunction: a case report. Cancer Chemother Pharmacol. 2014;73(2):429-433.

21. Stephenson JJ, Gregory C, Burris H, et al. An open-label clinical trial evaluating safety and pharmacokinetics of two dosing schedules of panitumumab in patients with solid tumors. Clin Colorectal Cancer. 2009;8(1):29-37.

22. Weiner LM, Belldegrun A, Rowinsky E, et al. Updated results from a dose and schedule study of Panitumumab (ABX-EGF) monotherapy, in patients with advanced solid malignancies. Proc Am Soc Clin Oncol. 2005;23:206.

23. Malik I, Hecht JR, Patnaik A, et al. Safety and efficacy of panitumumab monotherapy in patients with metastatic colorectal cancer (mCRC). $J$ Clin Oncol. 2005;23(16):3520. 
24. Van Cutsem E, Peeters M, Siena S, et al. Open-label phase III trial of panitumumab plus best supportive care compared with best supportive care alone in patients with chemotherapy-refractory metastatic colorectal cancer. J Clin Oncol. 2007;25(13):1658-1664.

25. Berlin J, Swanson P, Harker WG, Burris H, Hecht JR, Navale L. Panitumumab antitumor activity in patients (pts) with metastatic colorectal cancer (mCRC) expressing 10\% epidermal growth factor receptor (EGFr). J Clin Oncol. 2006;24(18S):3548.

26. Mitchell EP, Hecht JR, Baranda J, et al. Panitumumab activity in metastatic colorectal cancer (mCRC) patients (pts) with low or negative tumor epidermal growth factor receptor (EGFr) levels: an updated analysis. J Clin Oncol. 2007;25(Suppl 18):4082.

27. Fakih M, Wong R. Efficacy of the monoclonal antibody EGFR inhibitors for the treatment of metastatic colorectal cancer. Curr Oncol. 2010;17(Suppl 1):S3-S17.

28. Amado RG, Wolf M, Peeters M, et al. Wild-type KRAS is required for panitumumab efficacy in patients with metastatic colorectal cancer. J Clin Oncol. 2008;26(10):1626-1634.

29. Price TJ, Peeters M, Kim TW, et al. Panitumumab versus cetuximab in patients with chemotherapy-refractory wild-type KRAS exon 2 metastatic colorectal cancer (ASPECCT). A randomized, multicenter, open label, non-inferiority phase 3 study. Lancet Oncol. 2014;15(6): 569-579.

30. Peeters M, Price TJ, Cervantes A, et al. Randomized phase III study of panitumumab with fluorouracil, leucovorin, and irinotecan (FOLFIRI) compared with FOLFIRI alone as second-line treatment in patients with metastatic colorectal cancer. J Clin Oncol. 2010;28(31):4706-4713.

31. Hecht JR, Mitchell E, Chidiac T, et al. A randomized phase IIIB trial of chemotherapy, bevacizumab, and panitumumab compared with chemotherapy and bevacizumab alone for metastatic colorectal cancer. J Clin Oncol. 2009;27(5):672-680.

32. Douillard JY, Siena S, Cassidy J, et al. Randomized, phase III trial of panitumumab with infusional fluorouracil, leucovorin, and oxaliplatin (FOLFOX4) versus FOLFOX4 alone as first-line treatment in patients with previously untreated metastatic colorectal cancer: the PRIME study. J Clin Oncol. 2010;28(31):4697-4705.

33. Douillard JY, Oliner KS, Siena S, et al. Panitumumab-FOLFOX4 treatment and RAS mutations in colorectal cancer. $N$ Engl J Med. 2013; 369(11):1023-1034.

34. Heinemann V, von Weikersthal LF, Decker T, et al. FOLFIRI plus cetuximab versus FOLFIRI plus bevacizumab as first-line treatment for patients with metastatic colorectal cancer (FIRE-3): a randomised, open-label, phase 3 trial. Lancet Oncol. 2014;15(10):1065-1075.

35. Shankaran V. Cost Considerations in the evaluation and treatment of colorectal cancer. Curr Treat Options Oncol. 2015;16(8):41.

36. Kircher SM, Mohindra N, Nimeiri H. Cost estimates and economic implications of expanded RAS testing in metastatic colorectal cancer. Oncologist. 2015;20(1):14-18.

37. Tejpar S, Stintzing S, Ciardiello F, et al. Prognostic and predictive relevance of primary tumor location in patients with RAS wild-type metastatic colorectal cancer: retrospective analyses of the CRYSTAL and FIRE-3 Trials. JAMA Oncol. Epub 2016 Oct 10.

38. Venook AP, Niedzwiecki D, Innocenti F, et al. Impact of primary $\left(1^{\circ}\right)$ tumor location on overall survival (OS) and progression-free survival (PFS) in patients (pts) with metastatic colorectal cancer (mCRC): analysis of CALGB/SWOG 80405 (Alliance). J Clin Oncol. 2016;34:suppl, abstr 3504.

39. Allegra CJ, Rumble RB, Hamilton SR, et al. Extended RAS gene mutation testing in metastatic colorectal carcinoma to predict response-epidermal growth factor receptor monoclonal antibody therapy: American society of clinical oncology provisional clinical opinion update 2015. J Clin Oncol. 2016;342:179-185.

40. Hofheinz RD, Deplanque G, Komatsu Y, et al. Recommendations for the prophylactic management of skin reactions induced by epidermal growth factor receptor inhibitors in patients with solid tumors. Oncologist. 2016;21(2):1483-1491.
41. Burtness B, Anadkat M, Basti S, et al. NCCN Task Force Report: management of dermatologic and other toxicities associated with EGFR inhibition in patients with cancer. J Natl Compr Canc Netw. 2009; 7(Suppl 1):S5-S21.

42. Fabbrocini G, Panariello L, Caro G, Cacciapuoti S. Acneiform rash induced by EGFR inhibitors: review of the literature and new insights. Skin Appendage Disord. 2015;1(1):31-37.

43. Chanprapaph K, Vachiramon V, Rattanakaemakorn P. Epidermal growth factor receptor inhibitors: a review of cutaneous adverse events and management. Dermatol Res Pract. 2014;2014:734249.

44. Macdonald JB, Macdonald B, Golitz LE, LoRusso P, Sekulic A. Cutaneous adverse effects of targeted therapies: Part I: Inhibitors of the cellular membrane. J Am Acad Dermatol. 2015;72(2):203-218.

45. Lacouture ME, Anadkat MJ, Bensadoun RJ. Clinical practice guidelines for the prevention and treatment of EGFR inhibitor-associated dermatologic toxicities. Support Care Cancer. 2011;19(8):1079-1095.

46. Lacouture ME, Mitchell EP, Piperdi B, et al. Skin toxicity evaluation protocol with panitumumab (STEPP), a phase II, open-label, randomized trial evaluating the impact of a pre-emptive skin treatment regimen on skin toxicities and quality of life in patients with metastatic colorectal cancer. J Clin Oncol. 2010;28(8):1351-1357.

47. Van Cutsem E, Tejpar S, Vanbeckevoort D, et al. Intrapatient cetuximab dose escalation in metastatic colorectal cancer according to the grade of early skin reactions: the randomized EVEREST study. J Clin Oncol. 2012;30(23):2861-2868.

48. Petrelli F, Borgonovo K, Barni S. The predictive role of skin rash with cetuximab and panitumumab in colorectal cancer patients: a systematic review and meta-analysis of published trials. Target Oncol. 2013; $8(3): 173-181$.

49. Koukakis R, Gatta F, Hechmati G. Skin toxicity and quality of life during treatment with panitumumab for RAS wild-type metastatic colorectal carcinoma: results from three randomised clinical trials. Qual Life Res. 2016;25(10):2645-2656.

50. Thaler J, Karthaus M, Mineur L, et al. Skin toxicity and quality of life in patients with metastatic colorectal cancer during first-line panitumumab plus FOLFIRI treatment in a single-arm phase II study. BMC Cancer. 2012;12:438.

51. Melosky B, Leighl NB, Rothenstein J, Sangha R, Stewart D, Papp K. Management of egfr tki-induced dermatologic adverse events. Curr Oncol. 2015;22(2):123-132.

52. Califano R, Tariq N, Compton S, et al. Expert Consensus on the Management of Adverse Events from EGFR Tyrosine Kinase Inhibitors in the UK. Drugs. 2015;75(12):1335-1348.

53. Costa A, Teipar S, Prenen H, Van Cutsem E. Hypomagnesaemia and targeted anti-epidermal growth factor receptor (EGFR) agents. Target Oncol. 2011;6(4):227-233.

54. Jiang DM, Dennis K, Steinmetz A, et al. Management of epidermal growth factor receptor inhibitor-induced hypomagnesemia. a systematic review. Clin Colorectal Cancer. 2016;15(3):e117-e123.

55. O’Neil BH, Allen R, Spigel DR, et al. High incidence of cetuximabrelated infusion reactions in Tennessee and North Carolina and the association with atopic history. J Clin Oncol. 2007;25(24):3644-3648.

56. Chung $\mathrm{CH}$, Mirakhur B, Chan E, et al. Cetuximab-induced anaphylaxis and IgE specific for galactose-alpha-1,3-galactose. N Engl J Med. 2008; 358(11):1109-1117.

57. Saif MW, Peccerillo J, Potter VA. Successful re-challenge with panitumumab in patients who developed hypersensitivity reactions to cetuximab: report of three cases and review of literature. Cancer Chemother Pharmcol. 2009;63(6):1017-1022.

58. Langerak A, River G, Mitchell E, Cheema P, Shing M. Panitumumab monotherapy in patients with metastatic colorectal cancer and cetuximab infusion reactions: a series of four case reports. Clin Colorectal Cancer. 2009;8(1):49-54. 


\section{Publish your work in this journal}

OncoTargets and Therapy is an international, peer-reviewed, open access journal focusing on the pathological basis of all cancers, potential targets for therapy and treatment protocols employed to improve the management of cancer patients. The journal also focuses on the impact of management programs and new therapeutic agents and protocols on

patient perspectives such as quality of life, adherence and satisfaction. The manuscript management system is completely online and includes a very quick and fair peer-review system, which is all easy to use. Visit http://www.dovepress.com/testimonials.php to read real quotes from published authors.

Submit your manuscript here: http://www.dovepress.com/oncotargets-and-therapy-journal 